\title{
Role of Intratympanic Dexamethasone for Intractable Posterior Canal Benign Paroxysmal Positional Vertigo
}

\author{
Morales-Olavarría C \\ Sarría-Echegaray $P$ \\ Til-Pérez G \\ Carnevale $C^{*}$
}

\begin{abstract}
Background: Benign paroxysmal positional vertigo is a frequent diagnosed disorder, most of the patients are successfully treated with reposition maneuvers. In between $3-12.5 \%$ of these patients remain symptomatic. Recent studies support the use of intratympanic corticosteroid for intractable vertigo with promising results

Material and methods: Patients diagnosed with benign paroxysmal positional vertigo between June 2017 and December 2019 in a tertiary university hospital and in two private hospitals were included in the study and analyzed prospectively. They were treated and followed with repositioning maneuvers and intratympanic dexamethasone injections if the criteria was met.

Results: 4 out 72 patients included in the study developed criteria for intractable vertigo after at least 6 repositioning maneuvers. The posterior semicircular canal was affected in all cases, 3 out of 4 patients experienced symptom resolution, after two, four and five intratympanic dexamethasone injections respectively.

Conclusions: The use of intratympanic steroids to treat patients with refractory benign paroxysmal positional vertigo showed encouraging results. We believe a multicenter randomized clinical trial should be performed to assess the efficacy of intratympanic steroids in the treatment of this pathology.
\end{abstract}

Keywords: Tinnitus; paroxysmal positional vertigo; anti inflammatory.

Department of Otorhinolaryngology Head \& Neck Surgery, Son Espases University Hospital, Palma de Mallorca Spain. E-mail: claudio.carnevale.orl@gmail.com Phone: 


\section{INTRODUCTION}

Benign Paroxysmal Positional Vertigo (BPPV) is the most common type of vestibular disorder, characterized by episodic vertigo lasting for a few seconds induced by changes in the head position. The prognosis is good, with spontaneous recovery in approximately $20 \%$ of cases by one month and in up to 50 percent in three months ${ }^{1}$. Posterior semicircular canal (PSC) is the most common clinical variant, followed by horizontal and superior canals (HSC and SSC). Rarely BPPV may be bilateral, or affect more canals simultaneously ${ }^{2-4}$.Diagnosis is based on the characteristics of the nystagmus evoked by Dix Hallpike maneuver, used to explore the PSC and the SSC canals and Mc Clure maneuver or roll test for the HSC (Table 1). Treatment is based on different repositioning maneuvers, these are grounded on the two currently accepted etiopathogenic hypothesis, the cupulolithiasis and canalolithiasis theories ${ }^{5}$, both described by Schuknecht. The Epley (EM) and The Semont Maneuvers (SM) are for the posterior semicircular canal; the Gufoni and Lempert maneuvers, for the horizontal semicircular canal; the Yacovino maneuver for the superior semicircular canal. The particle replacement maneuvers are very effective, especially in the case of the posterior duct: the success rate is estimated in 90 to $95 \%$ with an average of 1 to 3 maneuvers ${ }^{3-8}$. The rate is lower in the case of the HSC $(85 \%)^{9,10}$, the SCC success rate is difficult to esti mate due to data scarcity. However, there are patients ( 3.5 to $12 \%$, depending on the series) ${ }^{11,12}$ in which the symptomatology and nystagmus persist despite the reiteration of the maneuvers, and others, where it recurs after the symptomatology has been resolved (around 25\%, especially in the first 6 to 12 months). Horii et al ${ }^{13}$ refers to these cases as intractable BPPV. In this scenario an imaging study by Magnetic Resonance Imaging (MRI) is mandatory to rule out inner ear or central nervous system abnormalities. Treatment of intractable BPPV is a challenge. Two surgical techniques have been described as the last therapeutic step after failure of repositioning maneuvers: 1) the section of the posterior ampullary nerve and 2) the occlusion of the posterior semicircular canal. The latter is considered the most appropriate for patients with intractable vertigo, since is less destructive and easier to perform than the posterior ampullary nerve section ${ }^{14}$. Several endocrinological and metabolic factors have been proposed in relationship with persistent BPPV and intractable forms: Vitamin D, estrogens, growth hormone, thyroid hormones and corticosteroids seem to act in bone metabolism and otoconial mineralization ${ }^{15}$. Additionally corticosteroids after interacting with the intracellular glucocorticoid receptors of the inner ear, produce important anti inflammatory effects and play a role in fluid and electrolyte regula-tion, improving homeostasis of the inner ear ${ }^{16}$. In several studies in which high resolution 3D MRI has been employed to study patients with intractable persistent BPPV, structural changes in the inner ear such as filling defects in the semicircular canals have been observed. These findings may indicate a narrowing of the semicircular canal suggesting an inflammatory process of the membranous labyrinth that could explain the failure of the repositioning maneuvers ${ }^{17,18}$. Based on these considerations, Perez et al ${ }^{19}$ published in 2016 the first study on the use of intratympanic (IT) corticosteroids for the treatment of persistent BPPV of PSC in 9 patients. Authors showed that after 2 sessions of IT methylprednisolone injections, combined with repositioning maneuvers, patients experienced symptoms improvement without recurrence in $78 \%$ of cases. Similar results have been reported by Kelkar et al ${ }^{20}$ two years later using IT dexamethasone in two patients. After reading these promising results, in 2017 we started treating intractable BPPV with IT dexamethasone injections combined with repositioning maneuvers. In this article we report our experience with four patients.

\section{MATERIALS AND METHODS}

All patients diagnosed with BPPV between June 2017 and December 2019 in a tertiary university hospital and in two private hospitals were included and analyzed prospectively. A total of 72 patients were included. The high clinical suspicion based on the symptoms referred by the patients was the key to diagnose the BPPV. In every case a complete otoneurological examination was performed, including otomicroscopy, tuning forks, audiometry if needed, exploration of spontaneous nystagmus, head thrust, Romberg and Unterberger tests. Dix Hallpike maneuver was employed to explore the PSC and SSC and roll test for the HSC. PSC, HSC and SSC were involved in 64, 5 and 3 patients respectively. EM and SM were used for the PSC, the Yacovino maneuver for the SSC and Lempert maneuver for the HSC. During the first encounter with the patient, nystagmus was observed with the naked eye. Patients that presented with a difficult-to-explore nystagmus, doubts about its direction or poor response after one correctly executed maneuver, a videonystagmography was performed. According to

Table 1: Clinical characteristics according to the affected canal.

Canal affected

Posterior

Superior

Horizontal
Nystagmus

Vertical-torsional with the linear component of its fast phase directed upward (to the forehead) and the torsional one directed with the upper pole of the eye to the lower ear

Vertical-torsional with the linear component of its fast phase directed downward and the torsional one directed with the inferior pole of the eye to the contralateral lower ear

Geotropic (canalolithiasis) or apogeotropic (cupulolithiasis). The affected side is defined by the intensity of nystagmus.
Diagnostic Maneuver

Dix Hallpike

Dix Hallpike

Roll test 
the diagnostic protocol used in our department, all cases that required three or more maneuvers with complete resolution or not of the symptomatology, were studied with an MRI with gadolinium to rule out central causes of paroxysmal positional vertigo or inner ear abnormalities described in previous studies ${ }^{21}$. Patients that required six or more maneuvers were considered candidates for intratympanic injection of corticosteroids, as previously suggested by Perez et al ${ }^{19}$. In all cases a solution of $4 \mathrm{mg} /$ $\mathrm{ml}$ dexa-methasone was infused into the middle ear in the affected side through a tympanostomy tube previously placed under microscope and local anesthesia. A 25-gauge spinal needle was employed for this purpose. After each IT treatment, patients were told to avoid swallowing and to turn their head towards the non-affected side for 15 minutes (Figure 1). All patients initially had twoweekly injections of intratympanic dexamethasone, the intervals were increased progressively according to the symptoms and the vestibular examination. Before each dexamethasone injec-tion a $\mathrm{DH}$ maneuver was performed to confirm the persistence of BPPV, the following day a re-positioning maneuver was executed. Success was defined as the absence of symptoms and nystagmus during $\mathrm{DH}$ maneuver. Follow-up ranged from 15 to 23 months with a mean follow-up of 18.75 months ${ }^{20}$.

\section{RESULTS}

Four out of the 72 patients $(5.5 \%)$ were included in the study three of them were female and one was a male (Table 2). The age at diagnosis ranged from 44 to 83 years old, with a mean age of 61.75 years. Posterior semicircular canal was involved in all cases and no specific cause was identified in any of them. One case showed multicanal involvement of posterior and superior right semicircular canals. No bilateral involvement was observed. A gadolinium MRI was per-formed in all patients, no central nervous system or inner ear abnormalities were detected. One of the patients had a previous diagnosis of multiple sclerosis shown in the MRI. After six unsuccessful repositioning maneuvers, treatment with IT dexamethasone was conducted. A grommet was placed in the affected ear (3 in the right side and 1 in the left side) under local anesthesia in the outpatient clinic and IT dexamethasone was administered. Three patients experienced symptom resolution. Two, four and five IT injections were needed respectively. One patient experienced subjective clinical improvement, but nystagmus and vertigo persisted during $\mathrm{DH}$ maneuver after 8 IT dexamethasone injections. No complications or side effects were observed during treatment and none of the 3 cured patients experienced disease recurrence during the follow up period ${ }^{21}$.

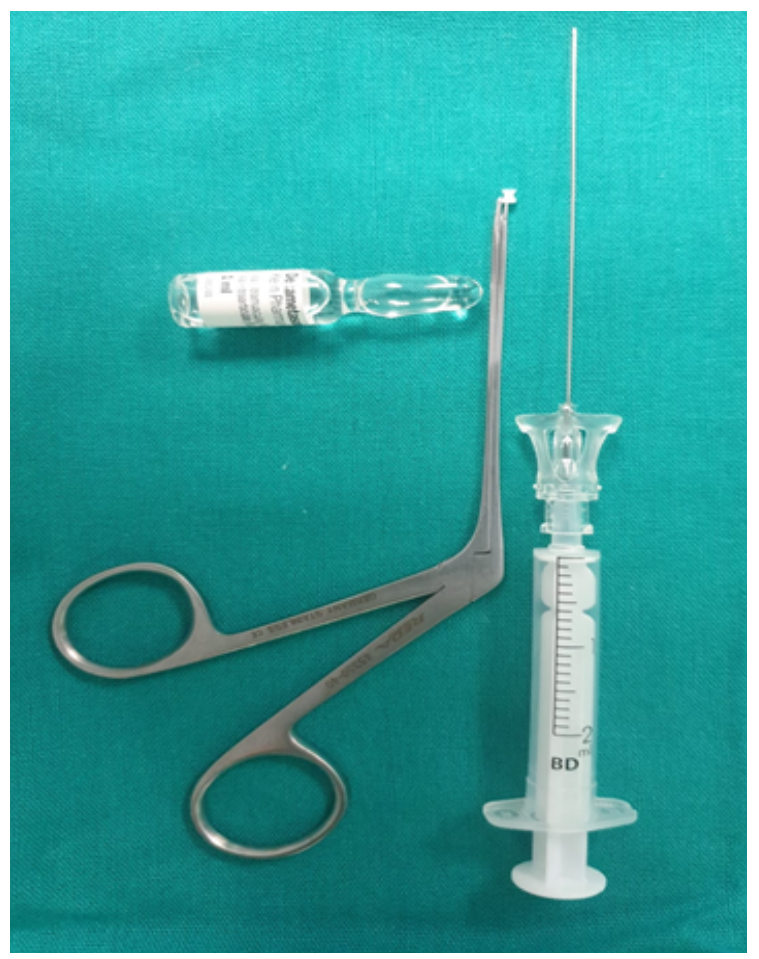

Figure 1: The frequency of concomitant diseases in high- and low-risk groups.

Table 2: Patients Treated with dexamethasone IT injections.

\begin{tabular}{cccc}
\hline Patient & Affected Canal & Number of IT injections & Symptom control \\
\hline 1 & Right posterior & 2 & Yes \\
2 & Left posterior & 4 & Yes \\
3 & Right posterior & 5 & Yes \\
4 & Right posterior and superior & 8 & No \\
\hline
\end{tabular}




\section{DISCUSSION}

BPPV is a common cause of vertigo. Most cases are idiopathic and respond successfully to canalicular repositioning maneuvers, others are self-limited and require no intervention ${ }^{22}$. A smaller group of patients experience persistent symptoms that don't resolve after multiple repositioning maneuvers (persistent vertigo), or recur after the symptomatology has been resolved (recurrent vertigo). The pathological bases remain unclear although several risk factors have been considered. All patients that belong to either of these two groups should be assessed with a neuro-radiological study to rule out central nervous system conditions that can mimic a BPPV before considering other factors. Although a specific cause is rarely identified, age and female sex seem to be intimately related with the origin of BPPV since it is commonly observed in female patients older than 60 years old. In a large series of patients with BPPV published by the authors, $6.25 \%$ of patients were considered refractory to the treatment, the presence of previous otoneuro logic disorders such as vestibular neuritis and multiple canal involvement were strongly associated with a higher risk of refractory BPPV 21 . Similar results were observed by Babac et al23. Age related vestibular degeneration and hormonal factors involved in changes in bone metabolism could play a role in the etiopathogenesis of BPPV ${ }^{24,25}$ and could explain some of the refractory cases. Different studies have identified osteoporosis/ osteopenia, estrogen deficiency, and low serum levels of vitamin $\mathrm{D}$ as potential risk factors for BPPV and higher risk of recurrence ${ }^{26}$. On the other hand, the possibility of multiple canal involvement in 4 to $21 \%$ of cases in different series ${ }^{27}$ and the fact that recurrent vertigo can affect any of the three canals in both labyrinths, suggest that some patients with BPPV do not suffer from a specific ailment in one of their inner ears, but rather develop a pathological condition involving their vestibular organs ${ }^{28}$. It is likely that a chronic inflammatory process of the inner ear or an alteration of the calcium sodium homeostasis of the labyrinth may be responsible for the refractory cases of BPPV. This hypothesis is suggested also by several findings observed in various studies where a threedimensional high-resolution MRI has been employed to study the inner ear of patients with refractory vertigo ${ }^{13}$. In these cases a higher incidence of abnormal MRI images (stenosis and canal filling defects) than normal controls have been reported. These anomalies might actually correspond to a ductal narrowness or to plugs of otoconial debris $^{13}$ that could be related to an inflammatory process or anomaly of calcium metabolism. It has been shown that glucocorticoid receptors are widely distributed throughout the inner ear, comprising the vestibular structures including the utricular macula, saccule and semicircular canal duct epithelium. Considering the potential role of gluco-corticoids receptors in the inner ear, IT injections of steroids could improve intractable vertigo through two possible pathways 1) anti-inflammatory properties and 2) modulation of the calcium metabolism. Based on these observations, Pérez et al. began to treat patients with intractable BPPV with intratympanic methylprednisolone and published their encouraging results in 2016. Steroids are largely employed to treat a great variety of inner ear disorders, such as Ménière's disease, autoimmune hearing loss and sudden idiopathic sensorineural hearing loss. IT steroid administration is a common procedure in the ENT outpatient clinic, it is usually well tolerated by the patient with a very low rate of complications. In our experience we have observed a higher treatment tolerance to the administration of dexamethasone than methylprednisolone, subsequently the first one was given to all patients. The decision to place a tympanostomy tube under microscope was based on the advantages of avoiding multiple punctures and being able to administer the drug with minor disturbance of the patient. In 3 out of 4 patients the symptomatology disappeared and showed no signs of recurrence throughout the followingup period. One patient showed no signs of improvement after multiple IT injections. In this case we identified a multiple canal involvement, a well described risk factor in the literature for persistent/recurrent BPPV ${ }^{28}$. Our results support the ones previously reported by Pérez et al in 2016 and by Kelkar et al two years later.

\section{CONCLUSION}

The use of IT steroids to treat patients with BPPV refractory to the maneuvers showed encouraging results in 3 out of 4 patients, with control of symptoms and improvement on quality of life during the follow-up. Although this study has several limitations, authors consider it is relevant to perform a multicenter randomized clinical trial to assess the efficacy of intratympanic steroids in the management of refractory BPPV.

\section{REFERENCES}

1. Burton MJ, Eby TL, Rosenfeld RM. Extracts from the Cochrane Library: modifications of the Epley (canalith repositioning) maneuver for posterior canal benign paroxysmal positional vertigo. Otolaryngol Head Neck Surg. 2012;147: 407-11.

2. Balatsouras DG. Benign paroxysmal positional vertigo with multiple canal involvement. Am J Otolaryngol. 2012;33:2508.

3. Carnevale C, Arancibia-Tagle D, Rizzo-Riera E, Til-Pérez G, Sarría-Echegaray P, Rama-Lopez J, et al. Efficacy of particle repositioning manoeuvres in benign positional paroxysmal vertigo: a revisión of 176 cases treated in a tertiary care centre. Acta Otorrinolaringol Esp. 2018;69:201-7.

4. Soto-Varela A, Rossi-Izquierdo M, Santos-Pérez S. Benign paroxysmal positional vertigo simultaneously affecting several canals: a 46-patient series. Eur Arch Otorhinolaryngol. 2012;270:817-22.

5. Steenerson RL, Cronin GW. Comparison of the canalith repositioning procedure and vestibular habituation training in forty patients with benign paroxysmal positional vertigo. Otolaryngol Head Neck Surg. 1996;114:61-4.

6. Epley JM. Human experience with canalith repositioning maneuvers. Ann N Y Acad Sci. 2001;942:179-91. 
7. Helminski JO, Zee DS, Janssen I, Hain TC. Effectiveness of particle repositioning maneuvers in the treatment of benign paroxysmal positional vertigo: a systematic review. Phys Ther. 2010;90:663-78.

8. Dorigueto RS, Ganança MM, Ganança FF. The number of procedures required to eliminate positioning nystagmus in benign paroxysmal positional vertigo. Rev Bras Otorrinolaringol (Engl Edition). 2005;71:769-75.

9. Brandt T, Huppert D, Hecht J, Karch C, Strupp M. Benign paroxysmal positioning vertigo: a long-term follow-up (6-17 years) of 125 patients. Acta Otolaryngol. 2006;126:160-3.

10. Kim JS, Oh SY, Lee SH. Randomized clinical trial for geotropic horizontal canal benign paroxysmal positional vertigo. Neurology. 2012;79:700-7.

11. Rupa V. Persistent vertigo following particle repositioning maneuvers: an analysis of causes. Arch Otolaryngol Head Neck Surg. 2004;130:436-39.

12. Choi SJ, Lee J B, Lim H J. Clinical features of recurrent or persistent benign paroxysmal positional vertigo. Otolaryngol Head Neck Surg. 2012;147:919-924.

13. Horii A, Kitahara T, Osaki Y, Imai T, Fukuda K, Sakagami $M$, et al. Intractable benign paroxysmal positioning vertigo: long-term follow-up and inner ear abnormality detected by three-dimensional magnetic resonance imaging. Otol Neurotol. 2010;31:250-5.

14. Maas BDPJ, van der Zaag-Loonen $\mathrm{HJ}$, van Benthem PPG, Bruintjes TD. Effectiveness of canal occlusion for intractable posterior canal benign paroxysmal vertigo: a systematic review. Otolaryngology Head and Neck Surgery. 2020;162:40-9.

15. Guerra J, Devesa J. causes and treatment of idiopathic benign paroxysmal positional vertigo based on endocrinological and other metabolic factors. J of Otology. 2020;15:155-160.

16. Pondugula SR, Sanneman JD, Wangemann $P$, Milhaud PG, Marcus DC. Glucocorticoids stimulate cation absorption by semicircular canal duct epithelium via epithelial sodium channel. Am J Physiol Renal Physiol. 2004;286:1127-35.

17. Horii A, Kitahara T, Osaki Y, Imai T, Fukuda K, Sakagami M, et al. Intractable benign paroxismal positional vértigo: long term flow-up and inner ear abnormality detected by threedimensional magnetic resonance imagine. Otol Neurotol. 2010;31: 250-5.
18. Dallan I, Bruschini L, Neri E, Nacci A, Segnini G, Rognini $F$, et al. the role of high-resolution magnetic resonance in atypical and intractable benign paroxysmal positional vertigo: our preliminary experience. ORL J Otorhinolaryngol Relat Spec. 2007;69:212-7.

19. Pérez $P$, Franco $V$, Oliva $M$, López Escámez JA. A pilot study using intratympanic methylprednisolone for treatment of persistent posterior canal benign paroxysmal positional vertigo. J Int Adv Otol. 2016;12:321-5.

20. Kelkar A, Johnson I. A novel use of intratympanic dexamethasone for intractable posterior canal benign paroxysmal vertigo: report of two cases. J Laryngol Otol. 2018;132:1147-49.

21. Carnevale $C$, Til Pérez G, Arancibia Tagle $D$, Tomás Barberán $M$, Sarria-Echegaray $P$. Identification of Factors Related to Cases of Benign Paroxysmal Positional Vertigo Refractory to Canalicular Repositioning Maneuvers and Evaluation of the Need for Magnetic Resonance Imaging in their Management: Retrospective Analysis of a Series of 176 Cases. 2010;1:1-6.

22. Rupa V. Persistent vertigo following particle repositioning maneuvers: an analysis of causes. Arch Otolaryngol Head Neck Surg. 2004;130:436-9.

23. Babac S, Djeric D, Petrovic-Lazic M, Arsovic N, Mikic A. Why do treatment failure and recurrences of benign paroxysmal positional vertigo occur? Otol Neurotol. 2014;35:1105-10.

24. Liu DH, Kuo CH, Wang CT, Chiu CC, Chen TJ, Hwang DK, et al. Age related increases in benign paroxysmal positional vertigo are reversed in women taking estrogen replacement therapy: a population-based study in taiwan. Front. Aging Neurosci. 2017;9:140-4.

25. Ogun OA, Janky KL, Cohn ES, Büki B, Lundberg YW. Gender-based comorbidity in benign paroxysmal positional vertigo. PLoS One. 2014;9:10554-6.

26. Talaat, HS, Kabel AM, Khaliel LH, Abuhadied G, El-Naga $H A$, Talaat AS. Reduction of recurrence rate of benign paroxysmal positional vertigo by treatment of severe vitamin D deficiency. Auris Nasus Larynx. 2016;43:237-241.

27. Balatsouras DG. Benign paroxysmal positional vertigo with multiple canal involvement. Am J Otolaryngol. 2012;33:250-8.

28. Pérez $P$, Franco V, Cuesta $P$, Aldama $P$, Alvarez MJ, Méndez JC. Recurrence of benign paroxysmal positional vertigo. Otol Neurotol. 2012;33:437-43. 